\title{
The Effect of Intra-wound Vancomycin Powder Application in Reducing Surgical Site Infections After Total Hip Arthroplasty
}

\author{
Farzad Amuzadeh Omrani ${ }^{1}$, Mohammad Emami ${ }^{1}$, \\ Mohammadmahdi Sarzaeem¹, Reza Zarei ${ }^{2}$ and Ali Yeganeh ${ }^{3 *}$ \\ ${ }^{1}$ Assistant Professor of Orthopedic Surgery, Imam Hossein Hospital, \\ Shahid Beheshti University of Medical Sciences, Tehran, Iran \\ ${ }^{2}$ Resident of Orthopedic Surgery, Imam Hossein Hospital, \\ Shahid Beheshti University of Medical Sciences, Tehran, Iran \\ ${ }^{3}$ Associate Professor of Orthopedic Surgery, Rasoul Akram Hospital, \\ Iran University of Medical Sciences, Tehran, Iran . \\ DOI: http://dx.doi.org/10.13005/bbra/1914
}

(Received: 16 October 2015; accepted: 30 November 2015)

\begin{abstract}
Post-operative total hip arthroplasty surgical wound infections are associated with substantial morbidity, mortality, and economic burden. Intra-wound vancomycin powder application may prevent infections after hip surgery. The objectives of this study is to answer the question as to whether the application of 2 gram intra-wound vancomycin powder In patients undergoing total hip arthroplasty, lead to reduced rates of postoperative surgical site infections or not? From April 2013, 125 patients had consent prior to their hip surgery. These 125 patients had total hip arthtoplasty by three different surgeons at Imam Hossein hospital. All patients received either 2 grams of IV Cefazolin one hour prior to the incision or 1 gram of IV Vancomycin one hour prior to the incision in patient with a Penicillin allergy. Near the end of the procedure, the wound was thoroughly irrigated with normal saline. for All patients befor surgical wound closure 2 gram of Vancomycin powder was poured into the wound. Postoperatively, patients continued on intravenous antibiotics for twenty-four hours. All patients underwent routine postoperative surgical care including physical therapy, routine radiographs, and routine managment of pain medications. Laboratory tests include $\mathrm{Hb}, \mathrm{BUN}, \mathrm{Cr}$ and electrolits were done before surgery and postoperatively after 48 hours and 7 days. All patients were discharged within the first 3 days after surgery and follow-up at weeks 2 and 4 and one year after surgery was performed. During the course of follow up that has ranged from one months to one year, sixteen of patients experienced surgical site infection. 10 patients were lost to follow up. No allergic reactions or adverse outcomes were reported from the use of 2 gram of Vancomycin powder prior to closure. Although intra-wound application of Vancomycin is known to minimize infections in spinal surgery little previous studies reported if Vancomycin application will reduce surgical site infection in hip surgeries. This study demonstrates that intra-wound application of Vancomycin reduces surgical site infection in total hip arthroplasty without any adverse complications.
\end{abstract}

Key words: Intra-wound Vancomycin , Total Hip Arthroplasty, Surgical Site Infection.

Surgical site infections following hip surgery are associated with a high morbidity and mortality and economic burden ${ }^{1-3}$.

\footnotetext{
* To whom all correspondence should be addressed. Tel : 00989122187996;

E-mail: yeganeh471@yahoo.com
}

Surgical site infections in hip surgery are among the most common postoperative complications. To minimize this risk, several methods of prophylaxis before, during and after the surgery are done. Treatment of these infections often requires long-term antibiotic treatment, multiple surgical procedures and complex wound 
management. Performance and quality of life of the patient and family will be impressed. It was recently shown that the use of intra-wound vancomycin powder is significantly reducing the risk of infection without Acute and long-term associated complications. Vancomycin administered into the wound prior to wound closure can reduce the incidence of post-operative surgical site infection after hip surgery ${ }^{4}$.

It is Suggested that this method is cost effective and free of systemic side effects ${ }^{5}$ but potential complications include renal toxicity and life-threatening anaphylaxis ${ }^{6}$. Recent studies on intra-wound vancomycin application have reported different estimates of efficacy. Several observation studies suggested significant benefits ${ }^{9}$. The aim of our study is to evaluate whether or not the intrawound vancomycin application can reduce surgical site infections after hip surgery.

\section{MATERIALAND METHODS}

The informed consent was obtained for all patients before hip surgery. From April 2013 to May 2015, 125 patients aged 45 to 91 years undergoing total hip arthroplasty was performed by different surgeons at Imam Hossein hospital.

Preoperatively, all patients prepared with alcohol, followed by 3M(tm) Duraprep(tm) surgical solution( lodine povacrylex [0/7\%available Iodine] and Isopropyl alcohol, 74\% W/W) Patient preoperative skin prepration. Additionally all patient received either 2 grams of IV cefazolin one hour prior to the incision or 1 gram of IV vancomycin one hour prior to the incision in patient with a penicillin allergy, which is a standard systemic prophylaxis in these procedures. No patient had a known vancomycin allergy.48 patients were male, 77 were female. Five patients were revision surgeries. 20 patients needed wire using because of fracture through broaching. Near the end of surgery after component insertion the wound was irrigated with at least 2 liters of normal saline.

At this point, 2 gram of vancomycin powder was poured into the wound. Post operatively patient continued to receive 1 gram of IV cefazolin every 8 hours for a total of 24 hours. For the penicillin allergic patient, they received 1 gram of vancomycin every 12 hours for a total of
24 hours.

All patients underwent routine postoperative surgical care including physical therapy, routine radiographs and routine management of pain and Anticoagulant therapy.

Laboratory tests include $\mathrm{Hb}, \mathrm{BUN}, \mathrm{Cr}$ and electrolytes were done before surgery and postoperatively after 48 hours and 7 days.

All patients were discharged within the first 3 days after surgery and follow-up at weeks 2 and 4 and one year after surgery was performed.

\section{RESULTS}

The course of follow up ranged from one month to one year. 10 patients were lost to follow up.

2 patients died in first 2 months but they have any sign of surgical site infection or other complications of intra-wound vancomycin powder. Creatinine increase occurred in 3 patients, all of which were created as a result of dehydration and after sufficient hydration tests returned to initial normal levels.

\section{No dislocation was reported}

A total of 125 patients, including 48 men and 77 women were divided into two groups.

Case group included 56 patients who had received intra-wound vancomycin powder at the end of surgery and the control group consisted of 69 patients who had not received the powder.

2 groups in terms of mean age and gender distribution did not differ significantly. Statistical analysis showed a significant difference between the group receiving intra-wound vancomycin powder and the control group in terms of the incidence of postoperative surgical site infections . The statistical analysis showed that there is significant difference in average operation time between patient with and without surgical site infection Statistical analysis showed that the incidence of surgical site infection in patients who smoke are significantly higher than non-smokers The findings show that in people with diabetes, the incidence of surgical site infection was significantly higher than in non-diabetics. The average age of patients who had surgical site infection showed no significant difference with those that were not.

By dividing the patients into two age 
groups above 60 years and below 60 years between the two groups showed no significant difference in the incidence of surgical site infection.

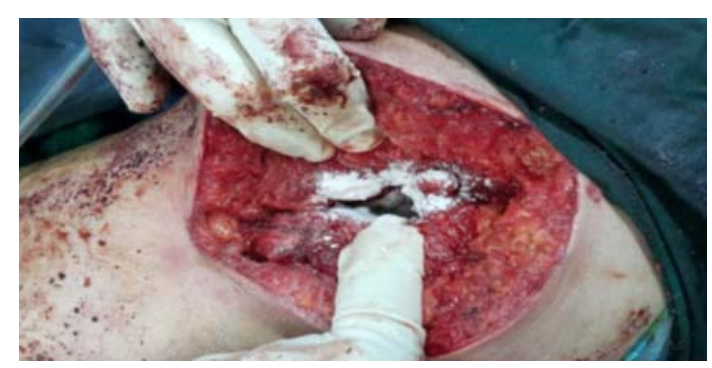

DISCUSSION

Reasons for higher rates of infection include longer operative time, prolonged retraction, and of course the use of foreign biomaterials ${ }^{9}$. The techniques that have been developed with aim at reducing the rate of infection following surgical procedures include generous irritation, debridement and extra effort at shorter operating times. Recently, a study by O'Neill showed that the application of intra-wound Vancomycin significantly reduces the risk of infection without any acute or long-term complications. (9) O’Neill et al reviewed 110 patients with traumatic spinal injuries undergoing instrumented spinal fusion procedures. Of the patients, 54received standard systemic prophylaxis, while 56 patients received standard systemic prophylaxis in addition to Vancomycin powder application in the surgical wound. The findings showed a significant decrease in the number of infections in the group treated with Vancomycin compared to the other group. This finding is very promising in decreasing the risk of postoperative surgical site infection and we anticipate that the use of intra-wound Vancomycin application will continue to increase. Previously, little studies were done to assess if intra-wound Vancomycin application will have any effects on reducing infection after total hip arthroplasty. Our study reveals that intra-wound Vancomycin application has significant effects on reducing surgical site infections and these findings are extremely valuable in the advancement of hip surgery. A study by Chen et al evaluated outcomes of treating infected tibial nonunion with Vancomycin impregnated cancellous bone grafting.(10) They followed-up 18 patients with infected tibial nonunion treated with adequate debridement, stabilized with external fixation, and staged Vancomycin-impregnated cancellous bone grafting. The group found that $100 \%$ of the patients were controlled adequately for infection, and bony union was achieved in 13 of the 18 patient after an average of 5.8 months. In the remaining 5 patients, bone union was achieved with close nailing 4 patients, and plating and bone grafting 1 patient.

They concluded that Vancomycinimpregnated cancellous bone grafting is an adequate treatment option for infected tibial nonunion. These findings are consistent with our results in terms of intra-wound Vancomycin powder application will reduce postoperative surgical site infection.

There are many studies showing the greater risk of complications and poorer clinical outcomes in patients undergoing revision hip surgeries. Revision surgeries for failure of total hip arthroplasty can cost the patient relevant time, money, and risks not to mention the emotional stress that surgical procedures can induce even after the recovery period.

Total hip arthroplasty surgery performed in a patient population with multiple co-morbidities must be approached very carefully and extra effort must be made to reduce infection, as additional surgeries may not be possible in chronically ill patients. Moreover, it has been shown that patients with previous history of hip surgery undergoing additional hip surgeries are more likely to develop infections.

Therefore, intra-wound Vancomycin application may be used to decrease infection rates in revision surgeries without causing any adverse complications as identified in this study.

Some of the limitations of this study include our Short term follow-up of patients, small sample size with our revision surgeries.

In conclusion, intra-wound application of Vancomycin powder is a very promising technique that will certainly be used more frequently to decrease the risk of infection in hip procedures and up until now, little studies have been done to assess this relationship.

The results of this study will enable surgeons to confidently use intra-wound Vancomycin for infection prophylaxis. We encourage more, larger sample size studies to be performed that not only look into this association, 
but also the relationship between other prophylactic measures and how they impact postoperative surgical site infection.

\section{REFERENCES}

1. Perencevich EN, Sands KE, Cosgrove SE, Guadagnoli E, Meara E, Platt R., Health and economic impact of surgical site infections diagnosed after hospital discharge. Emerg Infect Dis 2003; 9(2):196-203.

2. Kirkland KB, Briggs JP, Trivette SL, Wilkinson WE, Sexton DJ., The impact of surgical-site infections in the 1990s: attributable mortality, excess length of hospitalization, and extra costs. Infect Control HospEpidemiol 1999; 20(11): 725-730.

3. Fry DE., The economic costs of surgical site infection. Surg Infect (Larchmt) 2002; 3(Suppl1): S37-S43.

4. ZebalaL P, Chuntarapas T, Kelly MP, Talcott M, Greco S, Riew KD., Intrawound vancomycin powder eradicates surgical wound contamination: an invivo rabbit study. J Bone Jt Surg Am 2014; 96(1):46-51.

5. Godil SS, Parker SL, O’Neill KR, Devin CJ, Mc Girt MJ, Comparative effectiveness and costbenefit analysis of local application of vancomycin powder in posterior spinal fusion for spine trauma: clinical article. J Neurosurg Spine 2013; 19(3):331-335.

6. Rathbone CR, Cross JD, Brown KV, Murray CK, Wenke JC., Effect of various concentrations of antibiotics on osteogenic cell viability and activity. J Orthop Res 2011; 29(7):1070-1074.

7. Chiang HY, Herwald tL, Schweizer M., Reply to "letter to the editor" by baker and chen regarding "effectiveness of local vancomycin powder to decrease surgical site infections: A metaanalysis”. Spine $J$ [Epub ahead of print], 2014.

8. Debnath UK, Mehdian SM, Webb JK: Spinal Deformity Correction in Duchenne Muscular Dystrophy (DMD): Comparing the Outcome of Two Instrumentation Techniques. Asian Spine J 2011; 5:43-50.

9. O’Neill KR, Smith JG, Abtahi AM, Archer KR, Spengler DM, McGirt MJ, et al: Reduced surgical site infections in patients undergoing posterior spinal stabilization of traumatic injuries using vancomycin powder. Spine J 2011; 11:641-646, 09375534204.

10. Chen CE, Ko JY, Pan CC: Results of vancomycin-impregnated cancellous bone grafting for infected tibial nonunion. Arch Orthop Trauma Surg 2005; 125: 369-375. 